Analytical Chemistry • Supplementary Information

\title{
21-plex DiLeu isobaric tags for high-throughput quantitative proteomics
}

Dustin C. Frost ${ }^{\dagger}$, Yu Feng ${ }^{\dagger}$, and Lingjun $\mathrm{Li}^{\dagger, \neq^{*}}$

${ }^{\dagger}$ School of Pharmacy, University of Wisconsin-Madison, Madison, Wisconsin 53705, USA

${ }^{\ddagger}$ Department of Chemistry, University of Wisconsin-Madison, Madison, Wisconsin 53706, USA

*email: lingjun.li@wisc.edu 


\section{Contents:}

\section{Supplemental Methods}

Labeling efficiency calculation

\section{Supplemental Figures}

Figure S1 • 21-plex DiLeu reporter ion heavy isotope configurations

Figure S2 • Structures of new DiLeu isotopologues and isotopic reagents

Figure S3 • Chemical purity verification

Figure $\mathbf{S 4} \bullet$ Isotopic purity verification

Figure S5 • Measured isotopic purities

Figure S6 • Isotopic peak interferences to neighboring primary reporter ion signals

\section{Supplemental Tables}

Table S1 • Isotopic peak interferences to neighboring primary reporter ion signals

Table S2 • Channel mixing ratios for 15:10:5:1 samples 


\section{Supplementary Methods}

Labeling efficiency calculation. Following LC-MS/MS analysis of labeled samples, data is searched using Sequest HT in Proteome Discoverer 2.1 with both static and dynamic modification of the DiLeu tag on $\mathrm{N}$-termini and lysine to evaluate labeling efficiency. The \% labeling efficiency is determined by dividing the number of PSMs that contain the modification by the total number of PSMs reported. For example, a K562 tryptic digest sample was labeled with the $118 \mathrm{f}$ tag and subjected to LC-MS ${ }^{2}$ analysis on the Orbitrap Elite using the procedure and parameters specified in the Materials and Methods section. Following database search, 7515 PSMs were reported by the static mod search and 7578 PSMs were reported by the dynamic mod search, out of which 7419 were reported with the DiLeu tag mod, resulting in an estimated labeling efficiency of $98 \%$. Comparing the number of PSMs reported by the static vs. dynamic searches, the static search reported $99 \%$ of the number of PSMs reported by the dynamic search. 


\section{Supplementary Figures}
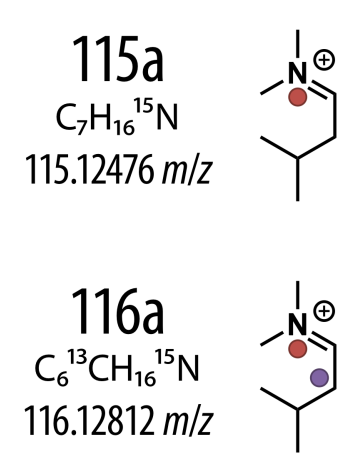

$\underset{116.13736 \mathrm{~m} / 2}{116 d}$
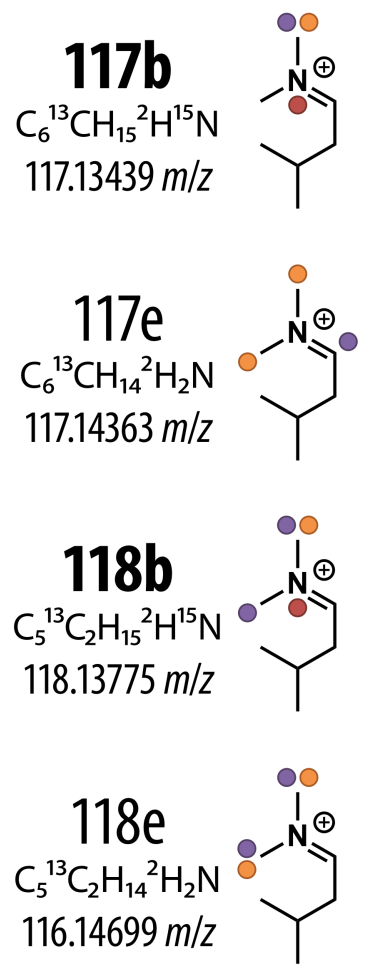
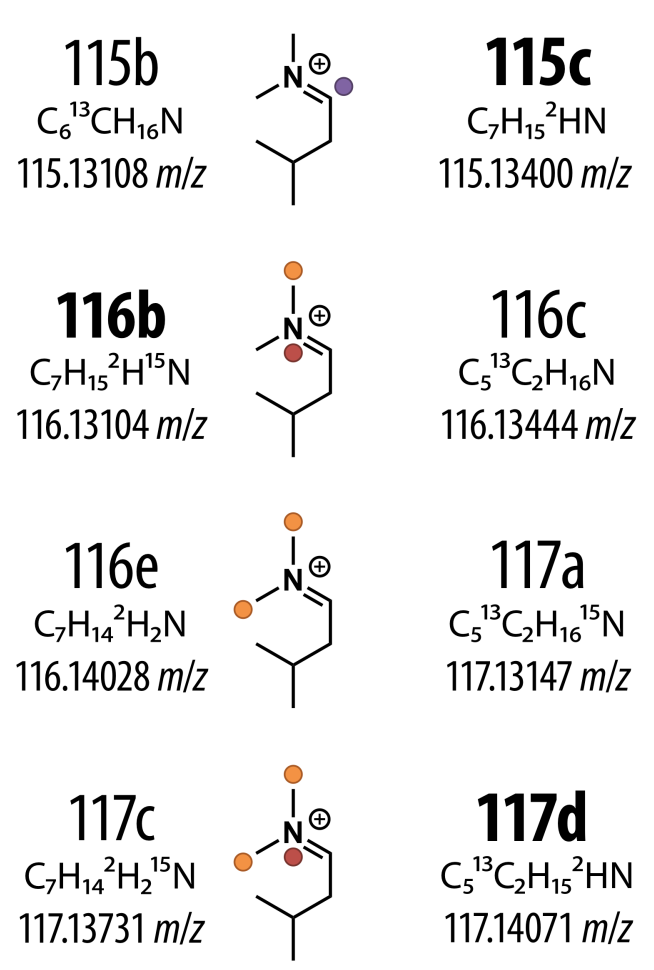

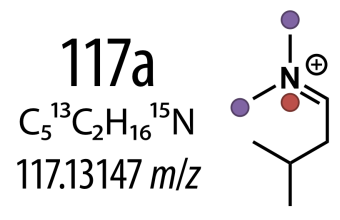

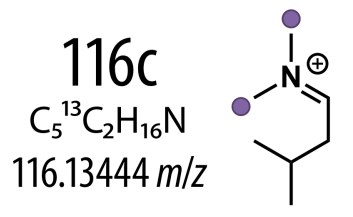

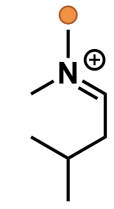

$115 \mathrm{C}$ $115.13400 \mathrm{~m} / \mathrm{z}$

$116.13444 \mathrm{~m} / \mathrm{z}$<smiles>CC(C)CC[N+](=O)[O-]</smiles><smiles></smiles><smiles>CC(C)CC=[N+](C)[O-]</smiles>

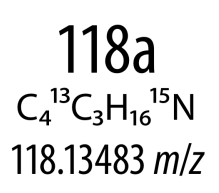

118d $\mathrm{C}_{7} \mathrm{H}_{13}{ }^{2} \mathrm{H}_{3}{ }^{15} \mathrm{~N}$ $118.14359 \mathrm{~m} / \mathrm{z}$<smiles>CC(C)CC[N+](=O)[O-]</smiles>

$118 \mathrm{~g}$ $\mathrm{C}_{7} \mathrm{H}_{12}{ }^{2} \mathrm{H}_{4} \mathrm{~N}$ $118.15283 \mathrm{~m} / \mathrm{z}$<smiles>CC(C)CC[N+](=O)[O-]</smiles>

${ }^{13} \mathrm{C} \quad{ }^{2} \mathrm{H} \quad{ }^{15} \mathrm{~N}$

Figure S1 - Heavy isotope configurations and exact masses $(\mathrm{m} / \mathrm{z})$ of 21-plex DiLeu reporter ions. New isotopologues are in bold. 

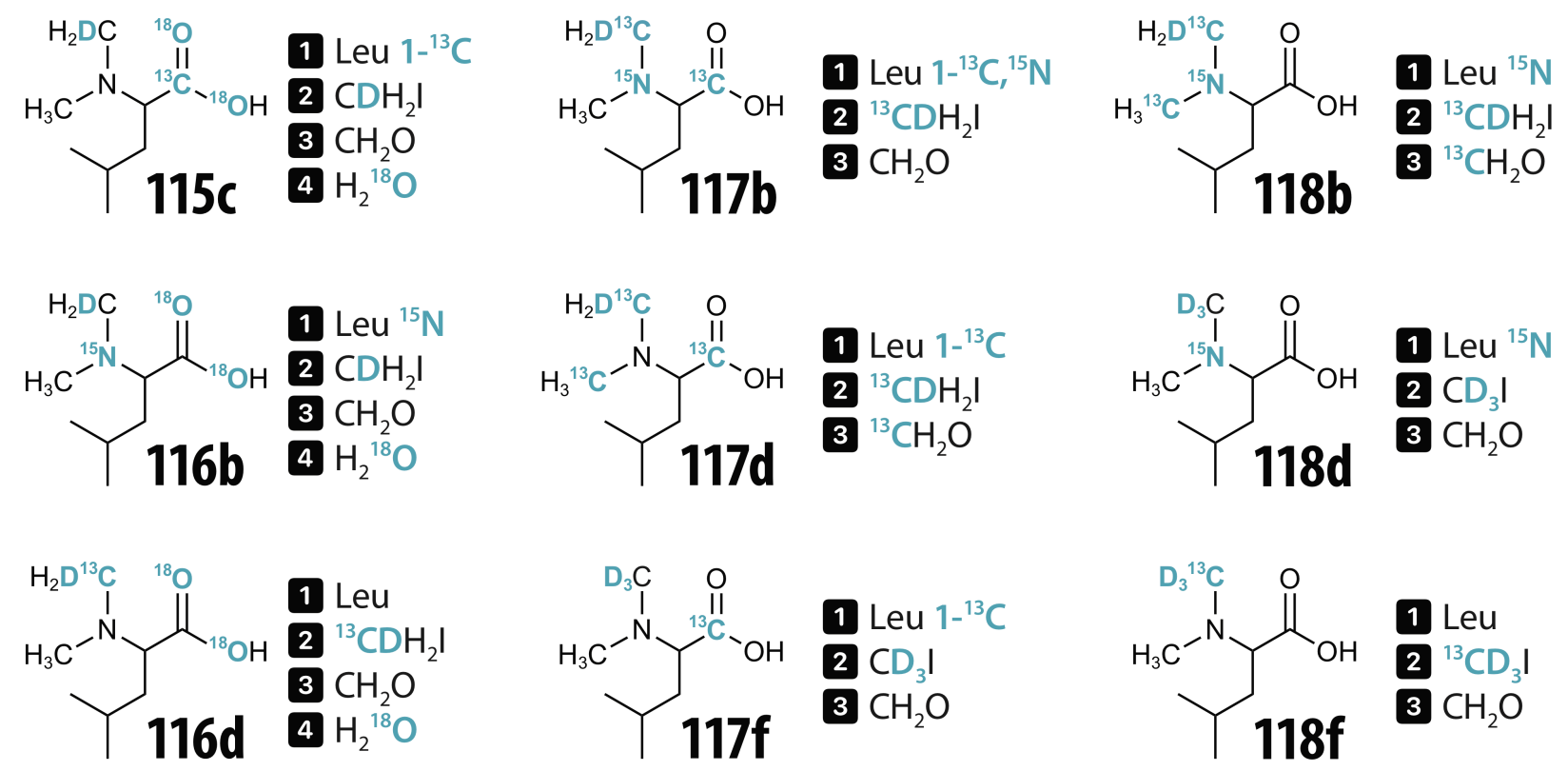

1 Leucine Cbz protection 2 lodomethane $N$-methylation $\quad 3$ Formaldehyde $N$-methylation $\quad \mathbf{4}{ }^{18}$ O exchange

Figure S2 - Structures of new DiLeu isotopologues and isotopic reagents used for synthesis. The order of isotope incorporation and choice of isotopic reagents were optimized for high isotopic purity. $\left(\mathrm{D}={ }^{2} \mathrm{H}\right)$. (1) $\mathrm{CbzCl}, 4 \mathrm{~N} \mathrm{NaOH}, 0{ }^{\circ} \mathrm{C}, 2 \mathrm{~h}$; (2) $\mathrm{NaH}, \mathrm{CH}_{3} \mathrm{l}$, THF, $0{ }^{\circ} \mathrm{C}, 16$ h; (3) $\mathrm{CH}_{2} \mathrm{O}, \mathrm{Pd} / \mathrm{C}, \mathrm{MeOH}: \mathrm{H}_{2} \mathrm{O}, \mathrm{H}_{2(\mathrm{~g})}, 80^{\circ} \mathrm{C}, 16 \mathrm{~h}$; (3) $\mathrm{HCl}_{(\mathrm{g})}$ in $\mathrm{H}_{2}{ }^{18} \mathrm{O}(\mathrm{pH} 1)$, $65^{\circ} \mathrm{C}, 4 \mathrm{~h}$. 


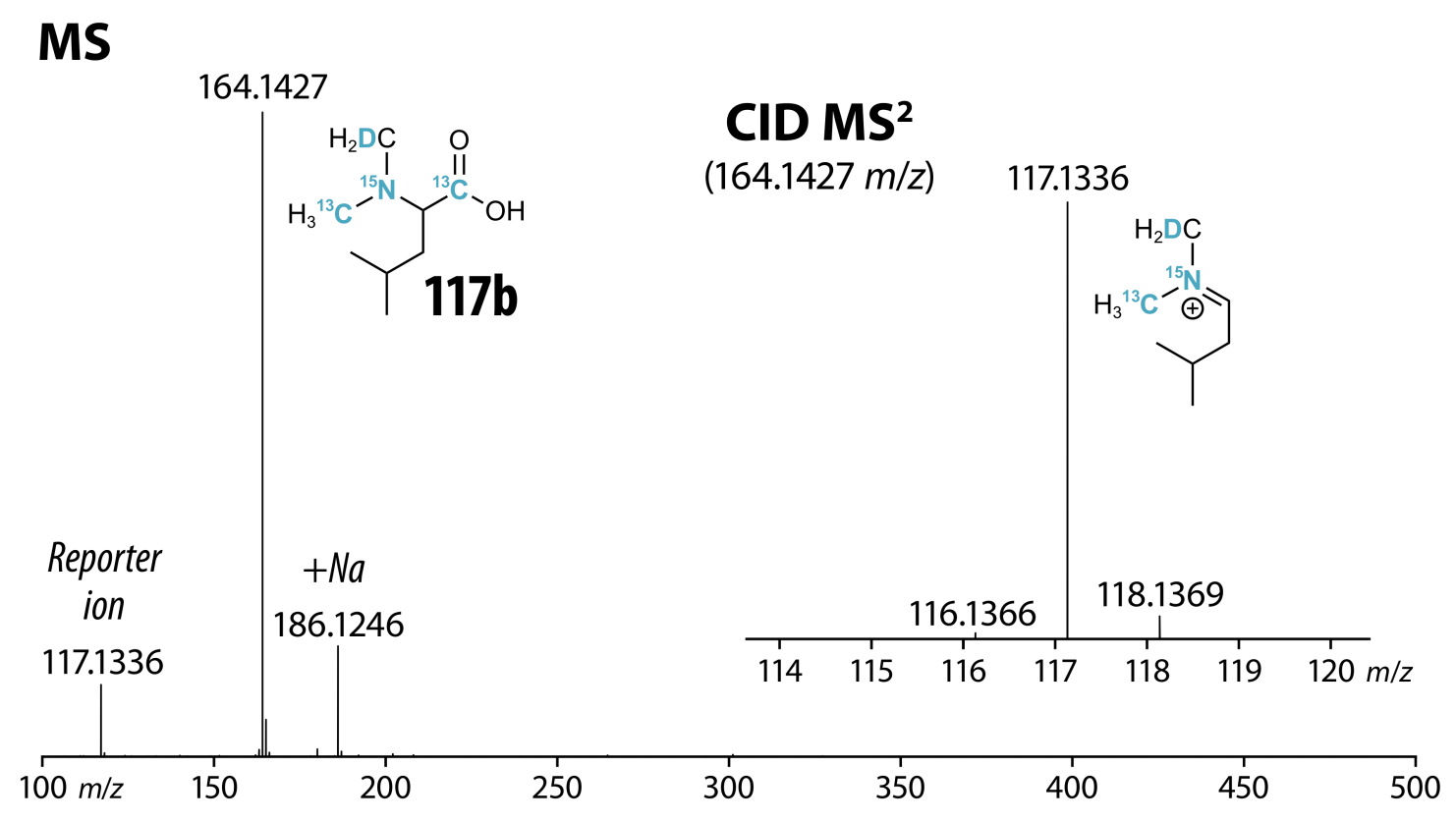

Figure S3 - Chemical purity verification. Representative example direct infusion MS spectrum of the synthesized DiLeu $117 \mathrm{~b}$ isotopologue is shown along with reporter ion CID MS ${ }^{2}$ spectrum. $\left(D={ }^{2} H\right)$ 

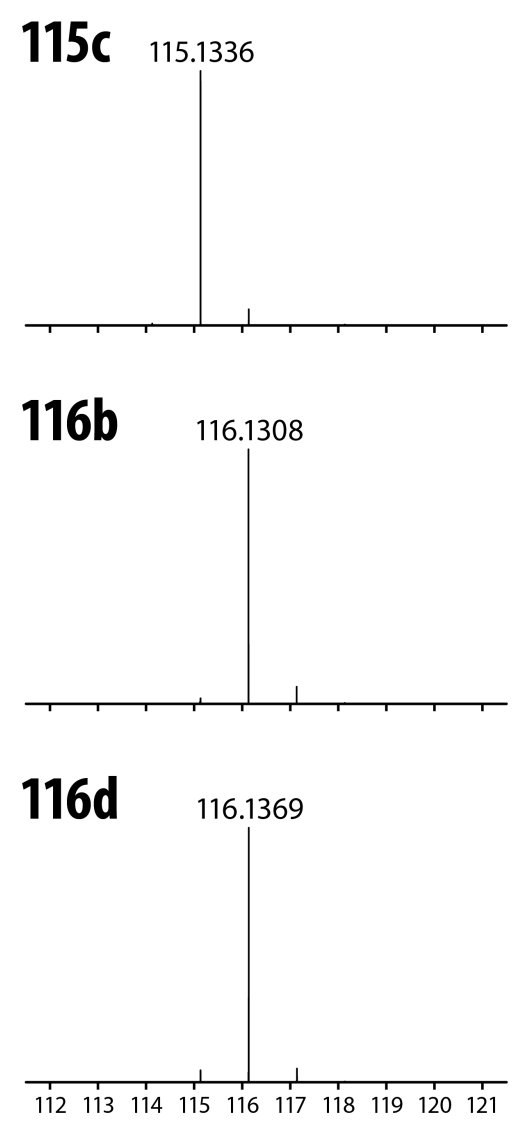
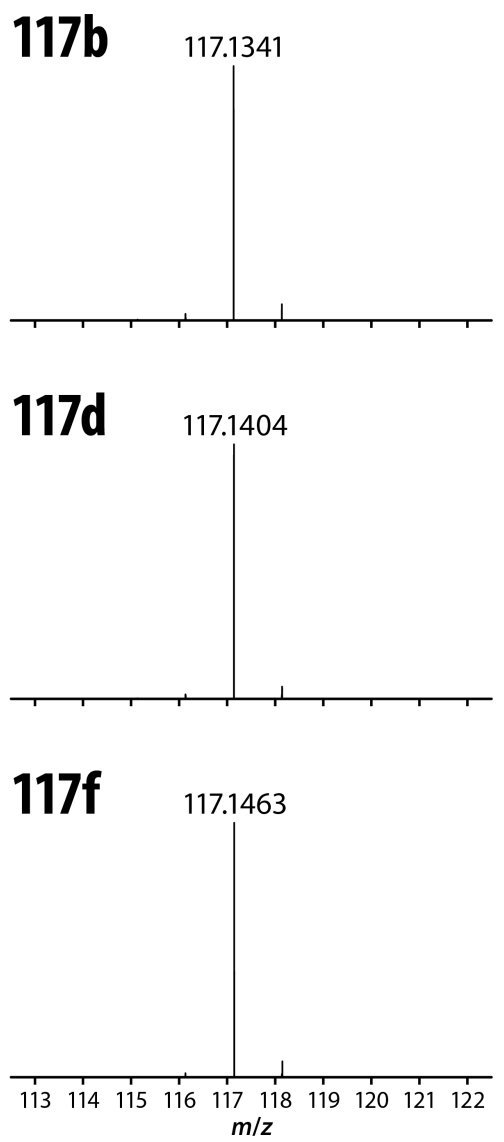
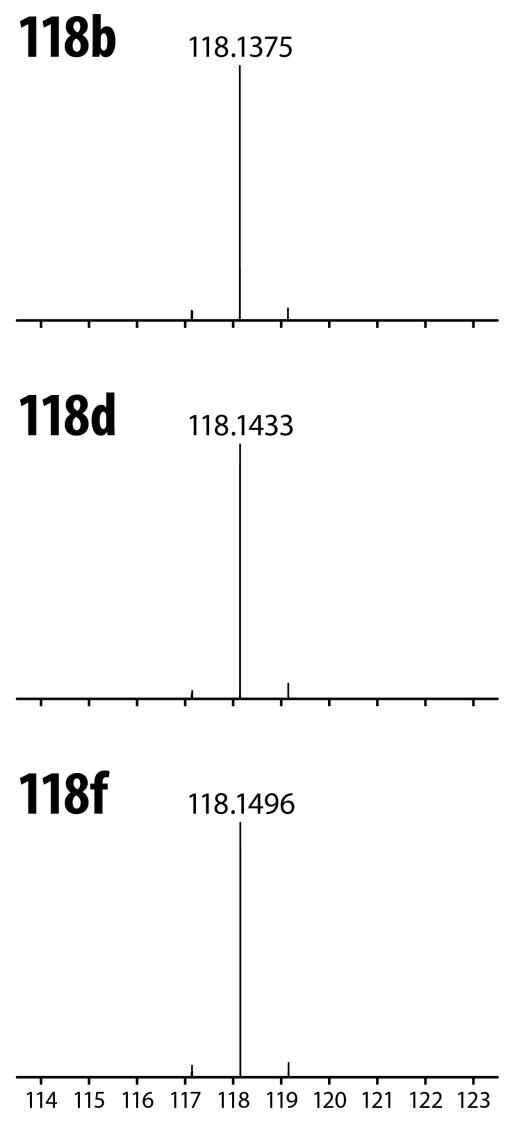

Figure S4 • Isotopic purity verification. Reporter ion CID MS ${ }^{2}$ spectra of each of the nine new $3 \mathrm{mDa}$ DiLeu isotopologues are shown. 


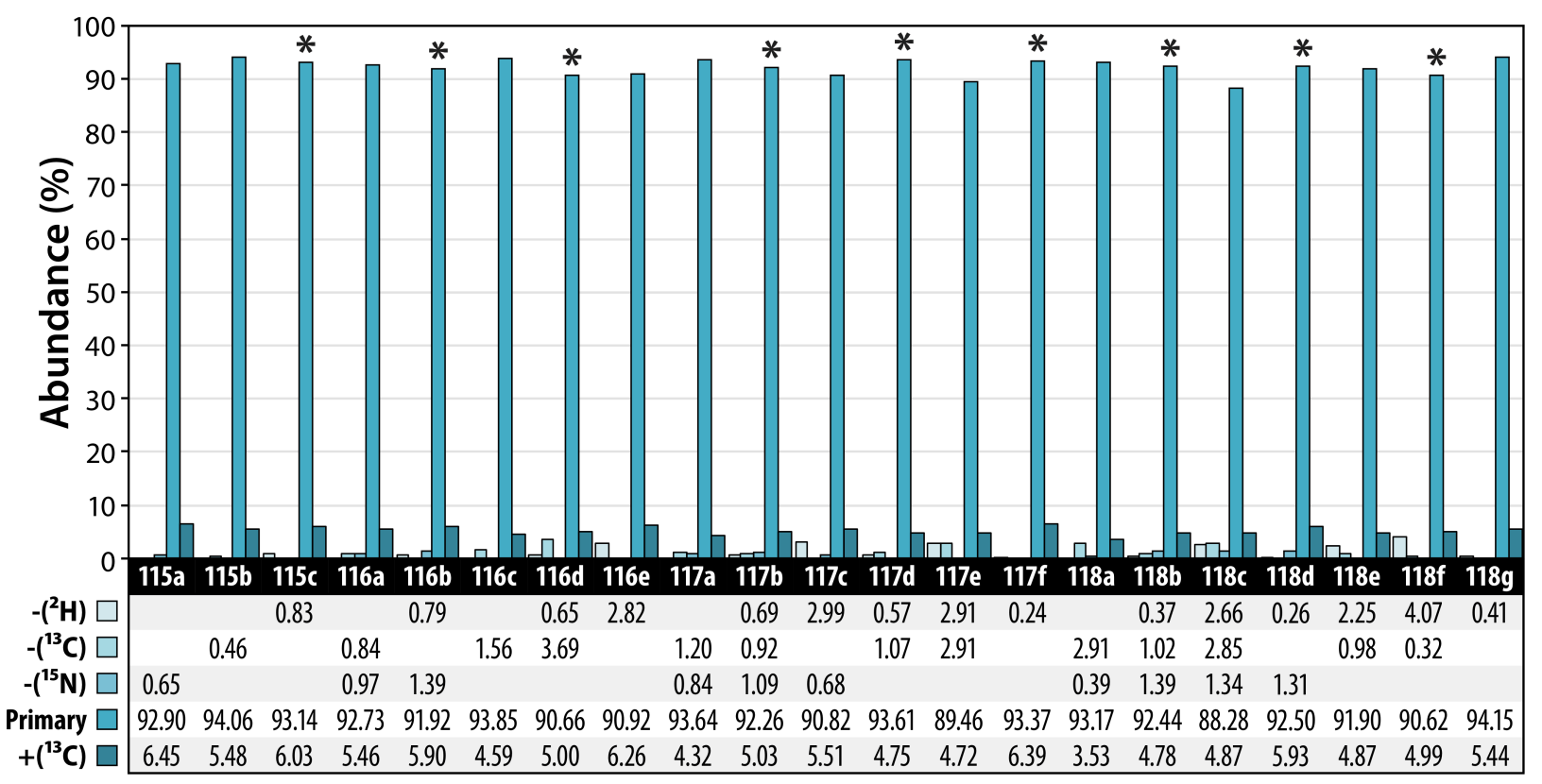

Figure S5 - Measured isotopic purities. 21-plex DiLeu isotopologues were subjected to direct infusion CID MS ${ }^{2}$ analysis to measure the fractional abundances (\%) of the -1 , primary, and +1 isotopic peaks for each channel; new $3 \mathrm{mDa}$ isotopologues are marked $\left.{ }^{*}\right)$. Isotopic purities may vary between synthesized batches and should be determined following each synthesis. 


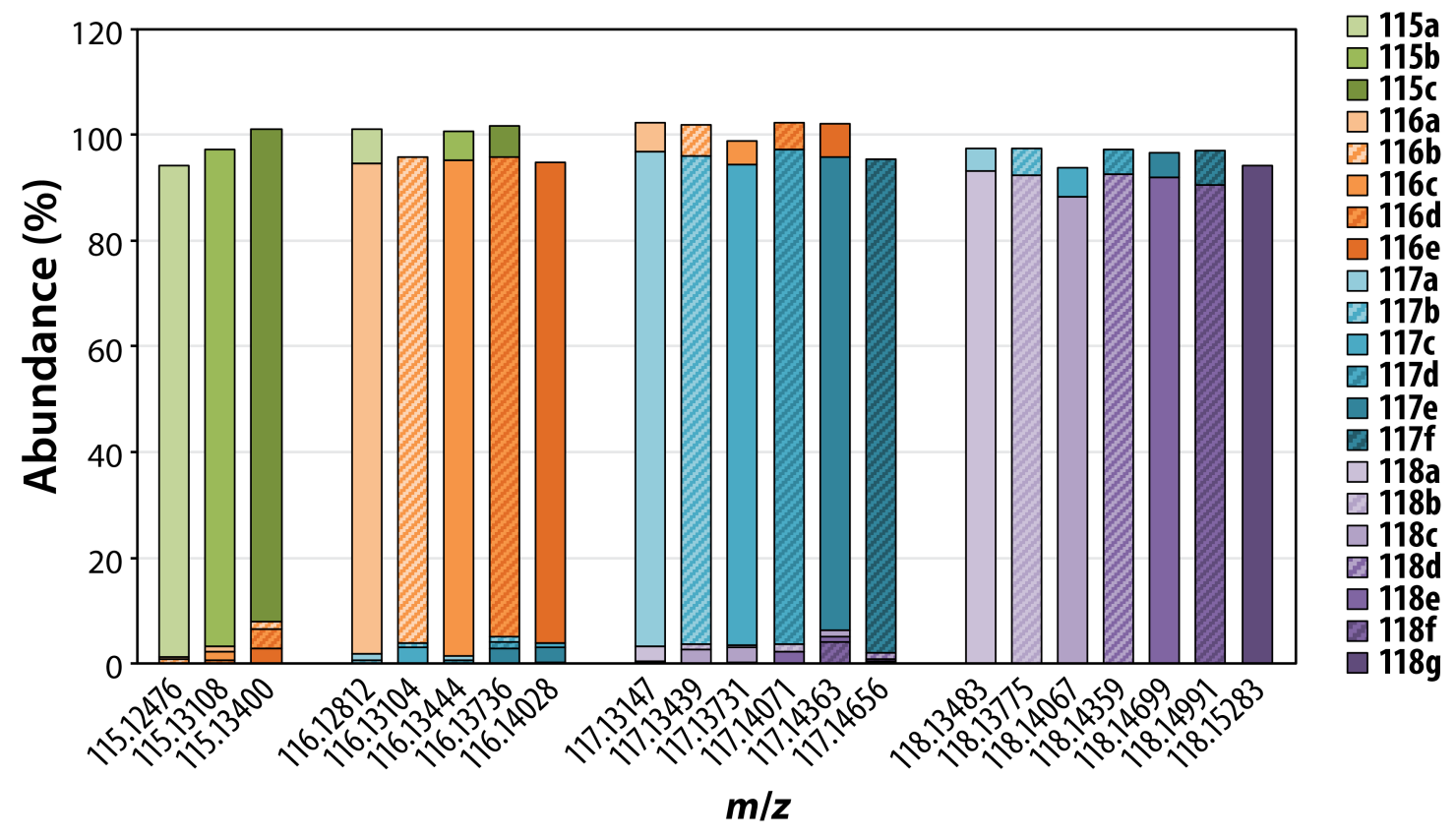

Figure S6 - Isotopic peak interferences to neighboring primary reporter ion signals. The fractional intensities of the $+1\left({ }^{13} \mathrm{C}\right)$ and $-1(\mathrm{H}, \mathrm{N}, \mathrm{C})$ isotopic peaks of each channel (in columns) overlap with neighboring primary reporter ion peaks (in rows) and contribute to the measured primary reporter ion intensities. 


\begin{tabular}{|c|c|c|c|c|c|c|c|c|c|c|c|c|c|c|c|c|c|c|c|c|c|}
\hline Primary $(\mathrm{m} / \mathrm{z})$ & $115 a$ & $115 b$ & $115 \mathrm{c}$ & $116 a$ & $116 b$ & $116 c$ & $116 d$ & $116 \mathrm{e}$ & $117 a$ & 117b & $117 c$ & 117d & 117e & $117 f$ & $118 \mathrm{a}$ & $118 b$ & $118 \mathrm{c}$ & 118d & $118 \mathrm{e}$ & $118 f$ & $118 \mathrm{~g}$ \\
\hline 115a (115.12476) & $92.90 \%$ & & & $0.84 \%$ & $0.79 \%$ & & & & & & & & & & & & & & & & \\
\hline 115b (115.13108) & & $94.06 \%$ & & $0.97 \%$ & & $1.56 \%$ & $0.65 \%$ & & & & & & & & & & & & & & \\
\hline 115c (115.13400) & & & $93.14 \%$ & & $1.39 \%$ & & $3.69 \%$ & $2.82 \%$ & & & & & & & & & & & & & \\
\hline 116a (116.12812) & $6.45 \%$ & & & $92.73 \%$ & & & & & $1.20 \%$ & $0.69 \%$ & & & & & & & & & & & \\
\hline 116b (116.13104) & & & & & $91.92 \%$ & & & & & $0.92 \%$ & $2.99 \%$ & & & & & & & & & & \\
\hline 116c (116.13444) & & $5.48 \%$ & & & & $93.85 \%$ & & & $0.84 \%$ & & & $0.57 \%$ & & & & & & & & & \\
\hline 116d (116.13736) & & & $6.03 \%$ & & & & $90.66 \%$ & & & $1.09 \%$ & & $1.07 \%$ & $2.91 \%$ & & & & & & & & \\
\hline 116e (116.14028) & & & & & & & & $90.92 \%$ & & & $0.68 \%$ & & $2.91 \%$ & $0.24 \%$ & & & & & & & \\
\hline 117a (117.13147) & & & & $5.46 \%$ & & & & & $93.64 \%$ & & & & & & $2.91 \%$ & $0.37 \%$ & & & & & \\
\hline 117b (117.13439) & & & & & $5.90 \%$ & & & & & $92.26 \%$ & & & & & & $1.02 \%$ & $2.66 \%$ & & & & \\
\hline 117c (117.13731) & & & & & & $4.59 \%$ & & & & & $90.82 \%$ & & & & $0.39 \%$ & & $2.85 \%$ & $0.26 \%$ & & & \\
\hline 117d (117.14071) & & & & & & & $5.00 \%$ & & & & & $93.61 \%$ & & & & $1.39 \%$ & & & $2.25 \%$ & & \\
\hline 117e (117.14363) & & & & & & & & $6.26 \%$ & & & & & $89.46 \%$ & & & & $1.34 \%$ & & $0.98 \%$ & $4.07 \%$ & \\
\hline $117 f(117.14656)$ & & & & & & & & & & & & & & $93.37 \%$ & & & & $1.31 \%$ & & $0.32 \%$ & $0.41 \%$ \\
\hline 118a (118.13483) & & & & & & & & & $4.32 \%$ & & & & & & $93.17 \%$ & & & & & & \\
\hline 118b (118.13775) & & & & & & & & & & $5.03 \%$ & & & & & & $92.44 \%$ & & & & & \\
\hline $118 \mathrm{c}(118.14067)$ & & & & & & & & & & & $5.51 \%$ & & & & & & $88.28 \%$ & & & & \\
\hline 118d (118.14359) & & & & & & & & & & & & $4.75 \%$ & & & & & & $92.50 \%$ & & & \\
\hline $118 \mathrm{e}(118.14699)$ & & & & & & & & & & & & & $4.72 \%$ & & & & & & $91.90 \%$ & & \\
\hline 118f (118.14991) & & & & & & & & & & & & & & $6.39 \%$ & & & & & & $90.62 \%$ & \\
\hline $118 \mathbf{g}(118.15283)$ & & & & & & & & & & & & & & & & & & & & & $94.15 \%$ \\
\hline
\end{tabular}

Table S1 $\bullet$ Isotopic peak interferences to neighboring primary reporter ion signals. The fractional intensities of the $+1\left({ }^{13} \mathrm{C}\right)$ and $-1(\mathrm{H}, \mathrm{N}, \mathrm{C})$ isotopic peaks of each channel (in columns) overlap with neighboring primary reporter ion peaks (in rows) and contribute to the measured primary reporter ion intensities. 
Channel: 115a 115b 115c 116a 116b 116c 116d 116e 117a 117b 117c 117d 117e 117f 118a 118b 118c 118d 118e 118f 118g

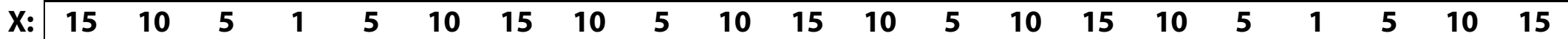

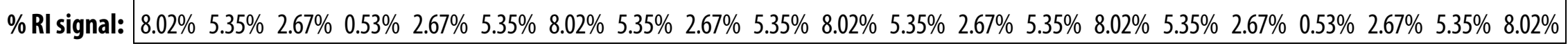

Table S2 • Channel mixing ratios $(X: 1)$ for 15:10:5:1 samples. The \% reporter ion signal refers to the fractional intensity of each individual channel in relation to the combined intensity of all channels $(100 \%)$. 